\title{
1 Mobile open dynamic chamber measurement of methane 2 macroseeps in lakes
}

3 Frederic Thalasso ${ }^{1}$, Katey Walter Anthony ${ }^{2,3}$, Olya Irzak ${ }^{4}$, Ethan Chaleff ${ }^{4}$, Laughlin Barker ${ }^{4}$, 4 Peter Anthony $^{2}$, Philip Hanke ${ }^{2}$, Rodrigo Gonzalez-Valencia ${ }^{1}$

$5 \quad{ }^{1}$ Biotechnology and Bioengineering Department, Cinvestav, Avenida IPN 2508, Mexico City, 07360, 6 Mexico

$7 \quad{ }^{2}$ Water and Environmental Research Center, University of Alaska Fairbanks, Fairbanks, Alaska 899775 , USA

9 International Arctic Research Center, University of Alaska Fairbanks, Fairbanks, Alaska 99775, 10 USA

$11{ }^{4}$ Frost Methane, Oakland, California 94612, USA

13 Correspondence to: Frederic Thalasso (thalasso@cinvestav.mx); Katey Walter Anthony 14 (kmwalteranthony@alaska.edu)

\section{Supporting Information:}

Number of pages: 6

Number of Figures: 6

Number of movie: 1

20 Number of Table: 1

21 Equations from $\mathrm{S} 1$ to $\mathrm{S} 6$

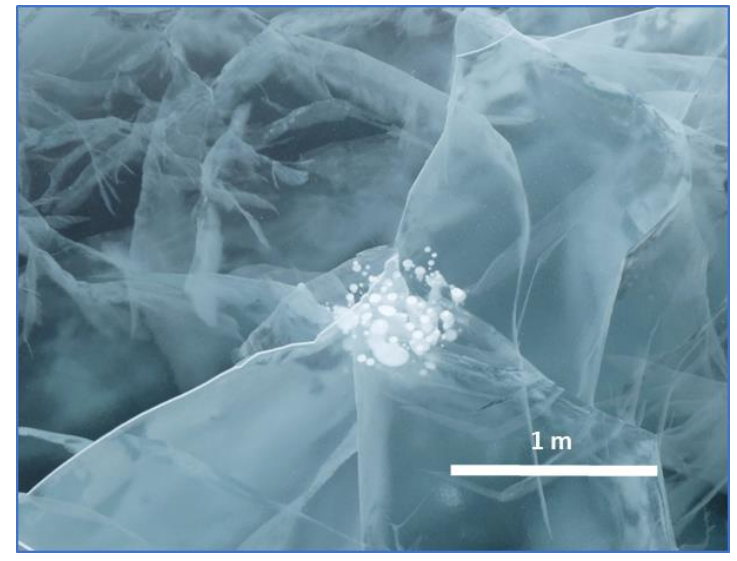

Figure S1. Methane bubbles trapped in the ice of an arctic lake, illustrating that ebullition occurs repeatedly in specific locations (Credit: A. Sepulveda-Jauregui, F. Thalasso). 
Figure S2. Dimension of the prototype built and used in the present work. Darker and lighter blue colors indicate three independent aluminum foils welded together. Dimensions are in $\mathrm{cm}$.

Movie S1. Methane seeps; general and closeup views. Available at: Thalasso, Frederic (2020), "Esieh lake seepage HESS", Mendeley Data, V1, doi: 10.17632/fnr3mkxmk9.1

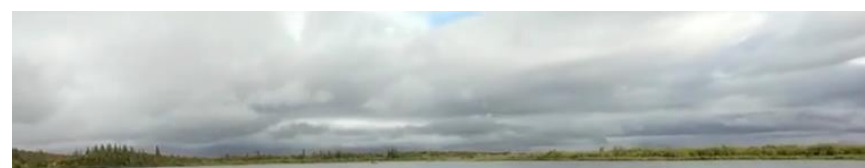

\section{S1. Response time and data interpretation}

The concentration read by the detector has a certain delay, due to the gas residence time from the chamber to the detector. However, if the detector is close to the chamber and the tubing of a reduced diameter, this time is very short; i.e., from 1.6 to $2.0 \mathrm{~s}$ in our case. However, even if it can be assumed that a bubble entering the chamber is immediately mixed within the chamber, the detectors have an inherent response time. This effect causes a certain delay and a buffer time, between the actual concentration read by the detector $\left(C_{D}\right)$ and $C_{C}$. To take this delay into account a standard mixing model can be used (Eq. S12), where $\theta$ is the response time of the system

$C_{C}=\left(\frac{d C_{D}}{d t} \cdot \theta\right)+C_{D}$

In Eq. (S1), $\theta$ was determined from experimental data, using several step $C_{D}$ increases observed in the field. The adjustment was done through excel, minimizing the Root Mean Square Error (RMSE) between experimental $C_{D}$ data and Eq. (S2), where $C_{D, 0}$ is the initial 55 reading of the detector (at time 0 ), and $C_{C}$ is the actual concentration in the chamber. 
$C_{D}=C_{D, 0}+\left[(1-\exp (-t / \theta)) *\left(C_{C}-C_{D, 0}\right)\right]$

After $C_{C}$ was determined, Eq. (5) was used to determine instantaneous $F$ along the transects. Alternatively, Eq. (6) was used to determined mean flux over a transect section. In the case of instantaneous F, during transects, and despite the relatively high signal to noise ratio of detectors used; i.e., ratio of the mean to the standard deviation, $F$ was subject to a significant noise, and a first data smoothening of $C_{C}$ was necessary, followed by a second smoothening of $d C_{C} / d t$ (Eq. S7). In both cases we opted for a pondered smoothening described by Eq. S3, where $\mathrm{X}^{\prime}$ is the smoothened variable $\mathrm{X}$, in this case $C_{C}$ or $d C_{C} / d t$.

$X_{t}^{\prime}=0.1 \cdot X_{t-2}+0.2 \cdot X_{t-1}+0.4 \cdot X_{t}+0.2 \cdot X_{t+1}+0.1 \cdot X_{t+2}$

As it will be shown in the Results and Discussion section, peak fluxes were detected, which corresponded to step increases of $C_{C}\left(\Delta C_{C}\right)$, caused by bubbles reaching the chamber. These abrupt increases offer a unique opportunity to quantify the $\mathrm{CH}_{4}$ mass content of the bubbles $\left(M_{B}\right)$. It should be noticed that since these step increases were observed in a few seconds, the amount of $\mathrm{CH}_{4}$ lost through detector extraction or entering the chamber can be neglected over that short time, as far a as single and clear increase was observed. Thus, $M_{B}$ was determined during the field experiment according to Eq. (S4).

$M_{B}=\Delta C_{C} \cdot V_{C}$

From $M_{B}$, the volume of the bubbles $\left(V_{B}\right)$ and their equivalent spherical diameter $\left(d_{B}\right)$ were determined, assuming that the $\mathrm{CH}_{4}$ content in the bubbles $(\% \mathrm{CH} 4)$ is known, according to Eq. (S5) and (S6), respectively.

81

$V_{B}=\frac{M_{B}}{16} \cdot \frac{R \cdot T}{P} \cdot \frac{1}{\%_{C H 4}}$

$84 \quad d_{B}=2 \cdot \sqrt[3]{\frac{3 \cdot V_{B}}{4 \cdot \pi}}$

where 16 is the molecular weight of $\mathrm{CH}_{4}(\mathrm{~g}), R$ is the universal gas constant $\left(\mathrm{L} \mathrm{atm} \mathrm{mol}^{-1}\right.$ 


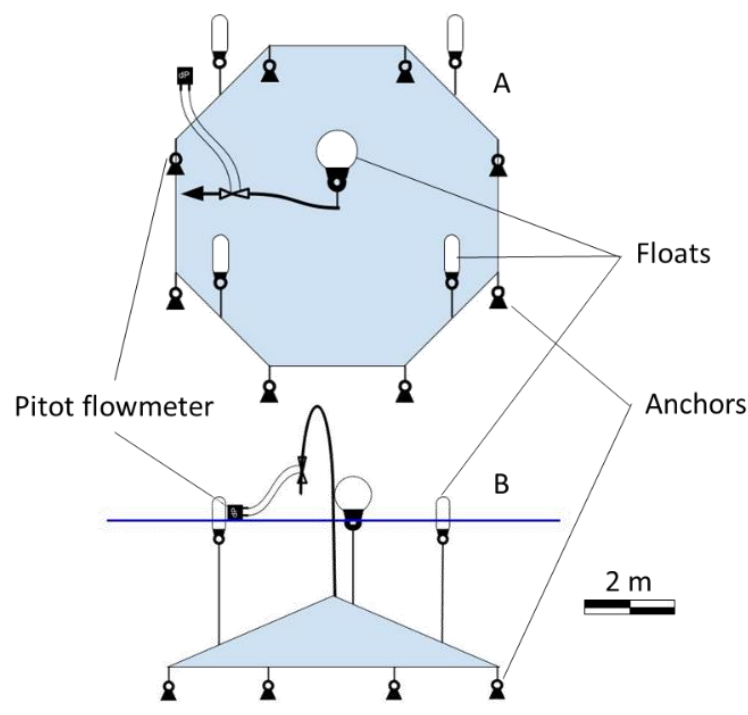

91

92 Figure S3. Conceptual sketch of the bubble trap used at Esieh Lake; (A) top view, (B) front 93 view.

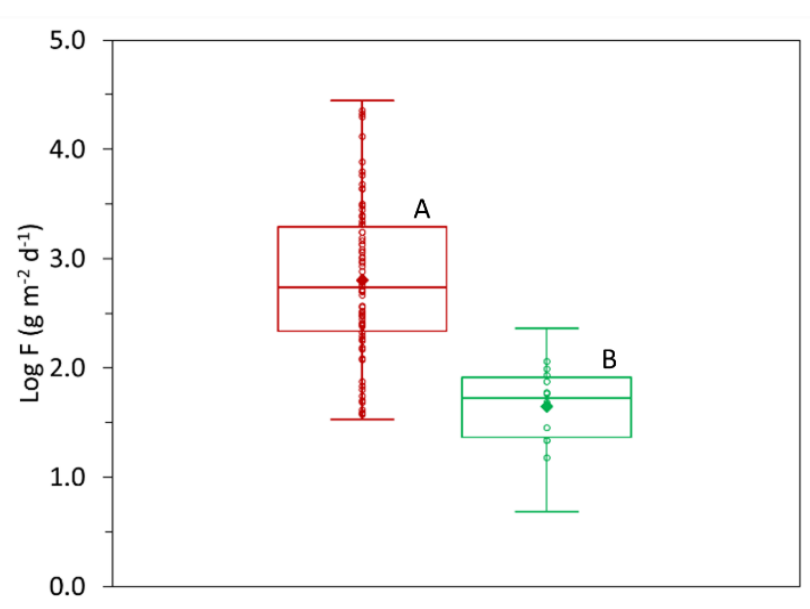

96 Figure S4. Box and whiskers showing statistical distribution of fluxes measured with the

97 MOD chamber (A, $n=74)$ and the diffusive component of these fluxes (B, $n=14$; see text 98 for details). Boxes show interquartile range and median, whiskers represent minimum and maximum, open circles show raw data and filled diamonds represent arithmetic mean. 


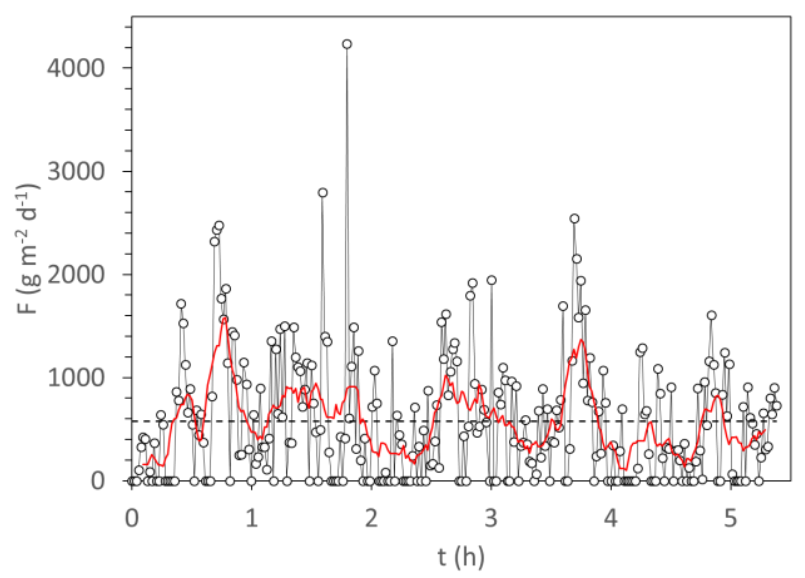

104 Figure S5. Flux measured by the bubble trap. Each discrete value is the average of 1 minute of continuous measurement. Horizontal discontinuous line shows the mean flux while red continuous line shows 10 minutes moving average of $F$ data.

107

108

109

110

111

112

113

114

115

116

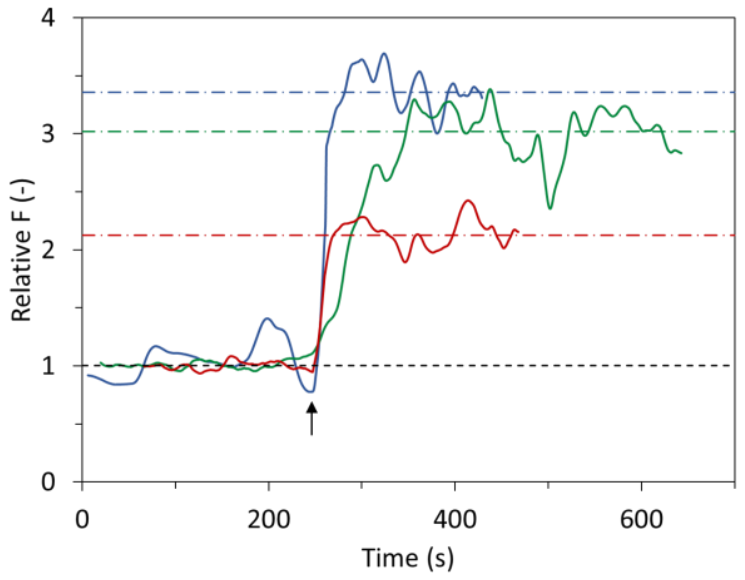

Figure S6: Relative fluxes observed with the MOD chamber, under stationary position (left of the arrows) and under motion. Data are presented in relative units, one being the flux observed in stationary position. Horizontal dot-dashed lines represent the mean fluxes during motion. 
118 Table S1: Comparison of four methods with a potential to be used in lake seepage.

\begin{tabular}{lllll}
\hline & Bubble trap & Duc et al. (2020) & Hydroacoustic & MOD Chamber \\
\hline Large seeps & Yes & Potentially Yes & Potentially Yes & Yes \\
Diffusive flux & No & Yes & No & Yes \\
Mobility & No & No & Yes & Yes \\
Autonomous & No & Yes & No & No \\
Field effort & Important & Moderate & Low & Low \\
Data processing effort & Low & Moderate & High & Moderate \\
Cost range (US\$) & Low-cost & Low-cost (un.) & $50000^{(1)}$ & $10000-50000^{(2)}$ \\
\hline
\end{tabular}

$119{ }^{1}$ Cost excluding video camera and mounting hardware; ${ }^{2}$ Cost of the detector (the cost of the 120 chamber assembly was about 300 US\$ in material). un.: undisclosed. 